\title{
Oral or parenteral antibiotic treatment in serious infections
}

When is it appropriate to use oral rather than parenteral antibiotics in the management of serious childhood infection? Intravenous infusion is used to induce high serum and tissue antibiotic concentrations rapidly in acutely ill patients, especially when large doses are needed, and is of course essential for children unable to tolerate oral medication. Nevertheless intravenous treatment is not without its drawbacks, not least the practical difficulties of controlling and supervising drug administration, the restriction imposed on children (most keenly felt during convalescence), and the possibility of cannula related sepsis; the widespread use of intravenous antibiotic treatment in paediatric wards has been questioned recently in the editorial column of this journal. ${ }^{1}$

Intravenous administration usually offers the quickest and most reliable means of establishing therapeutic antibiotic concentrations at the site of infection and avoids uncertainties related to incomplete absorption from the gastrointestinal tract. Intramuscular injection is unsuitable for large doses and impaired absorption may occur in patients with poor peripheral circulation. Once a clinical response to parenteral antibiotics has been established it becomes important to consider whether a child's interests are best served by changing to oral treatment-a decision that must be made in the light of clinical and microbiological aspects of the infection and of pharmacological properties of the antibiotic. Some of the problems are illustrated by the drugs and infections discussed below.

\section{Penicillins and cephalosporins}

Benzylpenicillin used in the treatment of bacterial meningitis should be given intravenously for a full 10 to 14 day course. It is well known that penicillins penetrate uninflamed meninges relatively poorly and that high serum concentrations must be maintained in convalescence when penetration into cerebrospinal fluid falls as the inflammatory process subsides. Therapeutic concentrations cannot be sustained by oral penicillin which is variably and incompletely absorbed from the gastrointestinal tract (only a quarter of an oral dose is recovered in urine). Treatment of meningococcal meningitis may be completed by an oral sulphonamide as long as the organism isolated is sulphonamide sensitive. Penicil- lin V may be used to complete courses for treatment of other infections initially controlled by benzylpenicillin where there is little chance of potentially damaging sequelae (pneumococcal pneumonia for example) and there is adequate penetration to the site of sepsis. It is important to ensure that the pathogens are fully sensitive and that doses are adequate; fortunately high doses of penicillins can be used in most patients without fear of toxicity. Absorption of penicillin $\mathrm{V}$ is increased as much as threefold if doses are taken one hour before meals ${ }^{2}$ and this property should be exploited. The host immune response plays an important role in eradicating most infections so it is perhaps wise to continue parenteral benzylpenicillin for a longer period in children with impaired immunity.

Initial treatment of staphylococcal osteomyelitis or septic arthritis usually includes intravenous flucloxacillin, commonly with fusidic acid as an adjunct because of its good penetration into bone and joints. In acute osteomyelitis, courses lasting four to 6 weeks are needed and the decision to change to oral treatment may be made once clinical assessment indicates that the infection is controlled: flucloxacillin rather than cloxacillin should be used because of its better absorption from the gastrointestinal tract. In patients with septic arthritis (who may be generally well) it is tempting to change to an oral penicillin at the earliest opportunity or even start treatment by mouth. Speedy and effective control of this infection is important, however, to avoid damage to the joint, and infection which has been active for some days before reaching medical attention should be treated vigorously. Penicillins achieve adequate concentrations in joint fluid $^{3}$ as long as sufficiently high doses are given (for example, $150 \mathrm{mg} / \mathrm{kg} /$ day flucloxacillin ${ }^{4}$ ) but penetrate joints less readily once inflammation has subsided. Dosage should not be reduced after an initial response to treatment; indeed some patients may need higher doses of flucloxacillin after changing from intravenous to oral treatment.

Ampicillin is incompletely absorbed after oral administration, some $30 \%$ of an oral dose being recovered in urine (compared with 60 to $70 \%$ after intramuscular injection). In bacterial meningitis caused by sensitive strains of Haemophilus influenzae high doses are required (150 to $200 \mathrm{mg} / \mathrm{kg}$ / day) and therapeutic concentrations in cerebro- 
spinal fluid must be maintained by intravenous administration. Oral ampicillin should not be used in this infection. Ampicillin concentrations in cerebrospinal fluid fall during convalescence, although this does not seem to influence the clinical outcome. ${ }^{2}$ Ampicillin is used widely for treatment of respiratory tract infections, though it penetrates relatively poorly into bronchial secretions; it achieves sputum concentrations about $10 \%$ of simultaneous serum concentrations after oral administration, though penetration may be two or three times greater in the presence of inflammation. It is important to use sufficiently high doses if oral ampicillin is used in treatment of lower respiratory tract infection-subtherapeutic concentrations not only lead to treatment failure but predispose to the development of bacterial resistance to ampicillin. Amoxycillin is absorbed twice as efficiently from the gastrointestinal tract and penetrates mucoid sputum more effectively than ampicillin, though penetration of both drugs into purulent bronchial secretion is similar. $^{2}$

Oral cephalosporins probably have a relatively minor role in the management of children with infection requiring hospital admission; they are best reserved for less serious infections in penicillin allergic children or in other cases where 'first line' antibiotics are not appropriate.

\section{Chloramphenicol}

Chloramphenicol is of course reserved for treatment of serious infections and although treatment may be given initially by the intravenous route, there are good reasons in many cases for giving the antibiotic by mouth as soon as a child is well enough to take oral medication. Peak concentrations are reached some two hours after an oral dose but exceed those after intravenous injection. Maximum serum concentrations after intramuscular injection are not reached for two to four hours and are about $50 \%$ of those obtained by equivalent oral doses. Some advise that the intramuscular route should be avoided wherever possible but satisfactory clinical results have, however, been obtained using intramuscular chloramphenicol (in Haemophilus influenzae meningitis, for example).

Chloramphenicol is usually given to children as the palmitate, which is rapidly and completely hydrolysed by pancreatic lipases followed by almost complete absorption. ${ }^{5}$ Nevertheless it should probably be avoided in neonates in whom there may be delayed and incomplete absorption as a result of deficient lipase activity. Chloramphenicol is given intravenously as the succinate, which is hydrolysed by liver enzymes at rates which vary considerably from person to person-between $6 \%$ and $73 \%$ is metabolised to biologically active compound. ${ }^{6}$ Rates of metabolism and excretion also vary between individuals and in serious infection it is often necessary to monitor serum chloramphenicol concentrations in order to maintain optimum dosage. ${ }^{7}$ This is especially important in neonates, both to ensure therapeutic drug concentrations and to avoid dose related toxicity. In older children there seems to be little reason for using parenteral chloramphenicol after the first few doses as long as the oral drug is tolerated; it is prudent to complete the course in hospital so that medication can be supervised. Concentrations of chloramphenicol in cerebrospinal fluid are higher than for any other antibiotic-30\% to $50 \%$ serum concentrations are achieved even in the absence of meningeal inflammation. A comparison of oral and intravenous chloramphenicol treatment of Haemophilus meningitis has shown that children given the antibiotic by mouth after two days' initial intravenous treatment responded as well as those who received intravenous chloramphenicol for the entire course. ${ }^{8}$ Chloramphenicol seems to be superior to ampicillin in controlling this infection and is also favoured because it avoids the need for prolonged parenteral treatment. Some have observed that marrow toxicity is more likely after oral than parenteral chloramphenicol but there is probably insufficient evidence to support this view. ${ }^{6}$

\section{Erythromycin}

Erythromycin may be given by mouth or intravenously: the intramuscular preparation is no longer used in the United Kingdom since it is painful and relatively small doses only can be given by this route. A number of formulations are available for oral use; the base is an unstable acid, variably absorbed giving relatively low serum concentrations, and enteric-coated tablets may also give unreliable absorption. The salt, erythromycin stearate and esters, and erythromycin estolate and succinate are also available. The estolate gives serum concentrations three to four times greater than base or stearate but as there is no persuasive evidence of its clinical superiority it is probably best avoided because of a greater likelihood of liver toxicity. Standard doses of oral erythromycin achieve concentrations high enough to treat infections caused by very sensitive organisms such as Streptococcus pneumoniae but the sensitivity of many strains of Haemophilus influenzae may be lower and therapeutic concentrations in some sites such as sinuses and respiratory secretions may not be achieved unless high oral doses or the intravenous route is used. For serious infections when 
erythromycin is indicated, therapeutic concentrations are most reliably and quickly achieved by intravenous administration using erythromycin lactobionate which produces considerably higher serum concentrations than the oral drug.

\section{Sodium fusidate}

Peak concentrations achieved two hours after an oral dose are high, there is considerable variation in the rate and amount absorbed, but accumulation occurs after repeated dosage. The drug is well distributed in the body, although it does not penetrate into cerebrospinal fluid. The intravenous route is probably necessary only for those unable to take oral medication and for fulminating infection (some cases of Staphylococcus aureus endocarditis, for example). In most patients it is best avoided because of its greater association with hepatotoxicity. ${ }^{9}$ It should of course be given with another antistaphylococcal agent such as flucloxacillin to suppress the emergence of mutants resistant to fusidic acid.

\section{Metronidazole}

Serum concentrations fully bactericidal to anaerobic bacteria are achieved after standard doses of metronidazole given orally or by rectal suppository. Peak concentrations are usually achieved between one and four hours after oral metronidazole but individual rates of absorption do vary. Absorption after rectal administration is slower and peak concentrations occur after four hours. Intravenous metronidazole may be needed to induce high serum concentrations immediately, for example in children undergoing surgery for perforated appendicitis, but treatment may be continued as early as possible by the oral or rectal routes. This is not only more simple but also considerably cheaper.

\section{Conclusions}

The issues may be complex as illustrated by the examples given here. Decisions about the best route for antibiotic administration must take into account the site and severity of infection, the known or likely pathogen, and its antibiotic susceptibility as well as the pharmacology of the antibiotic used. Discussion between clinician and microbiologist in the light of a clinical and laboratory evaluation of each case may often offer the best approach to resolving individual problems.

\section{References}

1 Anonymous. (editorial). Arch Dis Child 1983;58:161.

Kucers A, Bennett N,McK. The use of antibiotics. 3rd ed. London: William Heinemann Medical, 1979.

3 Nelson JD. Antibiotic concentrations in septic joint effusion. N Engl J Med 1971;284:349-53.

4 Garrod LP, Lambert HP, O'Grady F. Antibiotic and chemotherapy. 5th ed. Edinburgh: Churchill Livingstone, 1981.

5 Kauffman RE, Thirumoorthi MC, Buckley JA, et al. Relative bioavailability of intravenous chloramphenicol succinate and oral chloramphenicol palmitate in infants and children. $J$ Pediatr 1981;99:963-7.

6 Leitman PS. Oral chloramphenicol therapy (editorial). J Pediatr 1981;99:905-6.

${ }^{7}$ Mulhall A, deLouvois J, Hurley R. The pharmacokinetics of chloramphenicol in the neonate and young infant. $J$ Antimicrob Chemother 1983;12:629-39.

8 Tuomanen EL, Powell KR, Markes MI. Oral chloramphenicol therapy in treatment of $H$ influenzae meningitis. $J$ Pediatr 1981;99:986-74.

9 Humble MW, Eykyn SJ, Phillips I. Staphylococcal bacteraemia, fusidic acid and jaundice. $B r$ Med $J$ 1980;280:1495-8.

JANE SYMONDS

Russell's Hall Hospital, Dudley,

West Midlands DY1 2HQ 